\title{
A Controlled Pre-Post Analysis Investigating The Effect of Mindfulness On Perceived Stress and Cortisol Levels and Exam Performance in Medical Students
}

\author{
Jasmine Heath Hearn \\ Manchester Metropolitan University \\ Claire J Stocker ( $\square$ c.stocker@aston.ac.uk) \\ Aston University
}

\section{Research Article}

Keywords: medicine, medical school, meditation

Posted Date: August 24th, 2021

DOI: https://doi.org/10.21203/rs.3.rs-806427/v1

License: (c) (i) This work is licensed under a Creative Commons Attribution 4.0 International License. Read Full License 


\section{Abstract \\ Background.}

Medical students demonstrate higher levels of psychological distress compared with the general population and other student groups. Mindfulness interventions show promise in stress reduction for this group, and in the reduction of cortisol, an established clinical marker of the body's stress response. This study investigated the relationship of mindfulness to perceived stress, salivary cortisol and assessment performance in undergraduate medical students in response to stressful and non-stressful events.

\section{Methods.}

A controlled pre-post analysis design with within-groups comparisons. 67 medical students completed the Five Facet Mindfulness Questionnaire (FFMQ) and provided perceived stress ratings and saliva samples, from which cortisol was extracted, during group work (control/baseline) and immediately prior to end of year 2 examinations (experimental). Academic performance data was extracted for comparison with measures.

\section{Results.}

Salivary cortisol and perceived stress were significantly increased prior to examinations. The change in cortisol levels from baseline (non-stressful, group work conditions) to experimental (stressful, pre-exam conditions) showed a significant negative correlation with exam performance. FFMQ score showed a significant positive correlation with exam performance and a significant negative correlation with salivary cortisol concentration.

\section{Conclusions.}

This study suggests that there exists an important relationship between mindfulness and biomarkers of stress, which may manifest into improved assessment outcomes potentially through healthier, more adaptive coping and stress management strategies. A novel strength of the present study is the identification of individual facets of mindfulness associated with stress and exam performance (acting with awareness and non-judging) suggesting that these may be important targets for improving medical students' stress management and exam performance.

\section{Background}

The development of competent and compassionate doctors requires years of medical education. Unfortunately, some of the demands of medical training, including study burden, busy schedules [1] and the assessment process [2], demonstrate detrimental effects on the mental wellbeing of medical 
students. Recent studies estimate that the prevalence of depression, stress and anxiety in medical students is up to $30 \%, 49 \%, 32 \%$ respectively [3,4], which is higher than that of the general population and age-matched peers [5]. Excess stress in the short term may lead to sleeping disturbances and poorer academic performance [6], with pre-exam stress shown to predict academic performance in medical students [7], which may have further adverse effects on students' stress and wellbeing. Chronic stress may increase students' risk of cardiovascular disease, immune dysfunction, neuroendocrine dysregulation, and subsequent mental health disorders later in life [8]. Furthermore, psychological distress may be detrimental to empathy and optimism in medical students $[9,10]$, therefore demonstrating the need for effective stress management tools and support for this group of students, particularly in the early years of medical education.

In response to stressors, students often use maladaptive coping strategies such as escape avoidance, in which wishful thinking and behavioral efforts are made to escape or avoid the problem [11]. These avoidance behaviors have the potential to negatively affect academic performance [12]. In contrast, mindfulness is an approach-focused strategy to managing stress, involving non-judgmental awareness of the present moment, as opposed to avoiding negative experiences. Mindfulness-based interventions have been studied in medical students, with promising results in terms of stress reduction [13], and improvements in wellbeing [14]. Likewise, recent evidence has demonstrated that lower perceived stress was associated with greater mindfulness, and that a brief mindfulness intervention in medical training was effective in maintaining lower perceived stress [15]. Further, a recent systematic review concluded that mindfulness is beneficial for reducing stress in medical students [16], thereby demonstrating its efficacy for medical students.

Stressors act upon the Hypothalamus-Pituitary-Adrenal Axis (HPA axis) neuroendocrine pathway to secrete cortisol from the adrenal gland. Under normal physiological conditions, cortisol is released in a diurnal cycle, with highest plasma levels 30 minutes after waking each morning, reducing to an average level an hour after waking and remaining at that level for several hours before decreasing to a low [17]. However, cortisol concentrations show acute increases in response to stressors [18]. Glucocorticoid dysregulation is strongly associated with a wide range of stress factors, and under prolonged stress, cortisol can remain chronically elevated [19]. This causes damaging levels of inflammation in the central nervous system affecting neurological function [20] including hippocampal learning [21]. Increases in salivary cortisol before exams can inhibit both learning processes [22] and reduce exam performance [23]. This acute response makes cortisol is a useful clinical marker to determine physiological responses to stress [18]. Demonstrable reductions in cortisol following four days of mindfulness training have been observed in Thai medical students [24]. In contrast, a study in students from a range of faculties at Cambridge University found no relationship between plasma cortisol levels and mindfulness training [25]. However, the relationship between dispositional mindfulness, perceived stress, salivary serum cortisol as a reliable biomarker of stress, and academic performance has not been explored in medical students at the time of a stressful experience. This may have implications for the management of stress in medical students through mindfulness training before and during exam times and for student support services to help optimize wellbeing and academic attainment, thus providing a foundation for stress management in 
further clinical training. The present study, therefore, aimed to establish the potential role of mindfulness in medical student stress in response to stressful and non-stressful situations, and the impact of mindfulness on exam performance.

\section{Methods}

\section{Design}

This was a cross-sectional study with within- and between-subject assessments. Participants provided measures at baseline (non-stressful, group work condition), thereby acting as their own control, and prior to summative assessments (stressful condition) at the end of year 2 .

\section{Participants}

Eligible participants were undergraduate medical students recruited from three cohorts commencing Year 1 studies on three consecutive years (2014 to 2016). Inclusion criteria were: aged over 18 years of age (no upper age limit), students enrolled on an undergraduate medical training program, and were in their second year of study. All participants were introduced to mindfulness in Year 1 as part of their training and encouraged to adopt the practice, although this was entirely optional. Participation was voluntary, with written informed consent obtained, with 122 students participating in the study and 67 students completing all parts of the study (8 from the 2014 cohort, 40 from the 2015 cohort, and 19 from the 2016 cohort).

\section{Procedure}

At the end of Year 2, participants who consented were approached during a standard group work session in term time (non-stressful condition) and provided a saliva sample and a rating of their current stress level. After which a demographic questionnaire and the Five Facet Mindfulness Questionnaire-Short Form were administered. Following this, prior to summative assessments (stressful condition) at the end of terms Year 2, students provided another rating of their current stress level, and a saliva sample. Examinations were written short answer papers, based on 12 clinical vignettes, with a possible ten marks available per vignette. Scenarios are based upon common presentations a student would be expected to see in practice. Upon completion of the study, participants were provided with a debrief letter detailing the study and reminding them of their right to withdraw until data were anonymized for analysis.

\section{Measures}

\section{Demographic Characteristics}

Of the 67 participants that provided complete data (i.e. baseline and pre-exam measures of perceived stress and saliva samples), 27 (40.3\%) were male and 61 (91\%) were single. The sample was diverse in ethnicity; $28.4 \%$ of the sample were white, $44.8 \%$ were Asian, and $13.4 \%$ were black/Caribbean/African. Detailed participant characteristics can be found in table 1. Independent-samples t-tests were conducted 
at baseline to compare the influence of demographic characteristics on mindfulness, baseline stress and cortisol, pre-exam stress and cortisol, and exam results. There were no significant differences in gender across these outcomes $(p>.05)$. There were also no significant differences between those who had any previous experience of mindfulness and those who had none.

Table 1. Participant demographics.

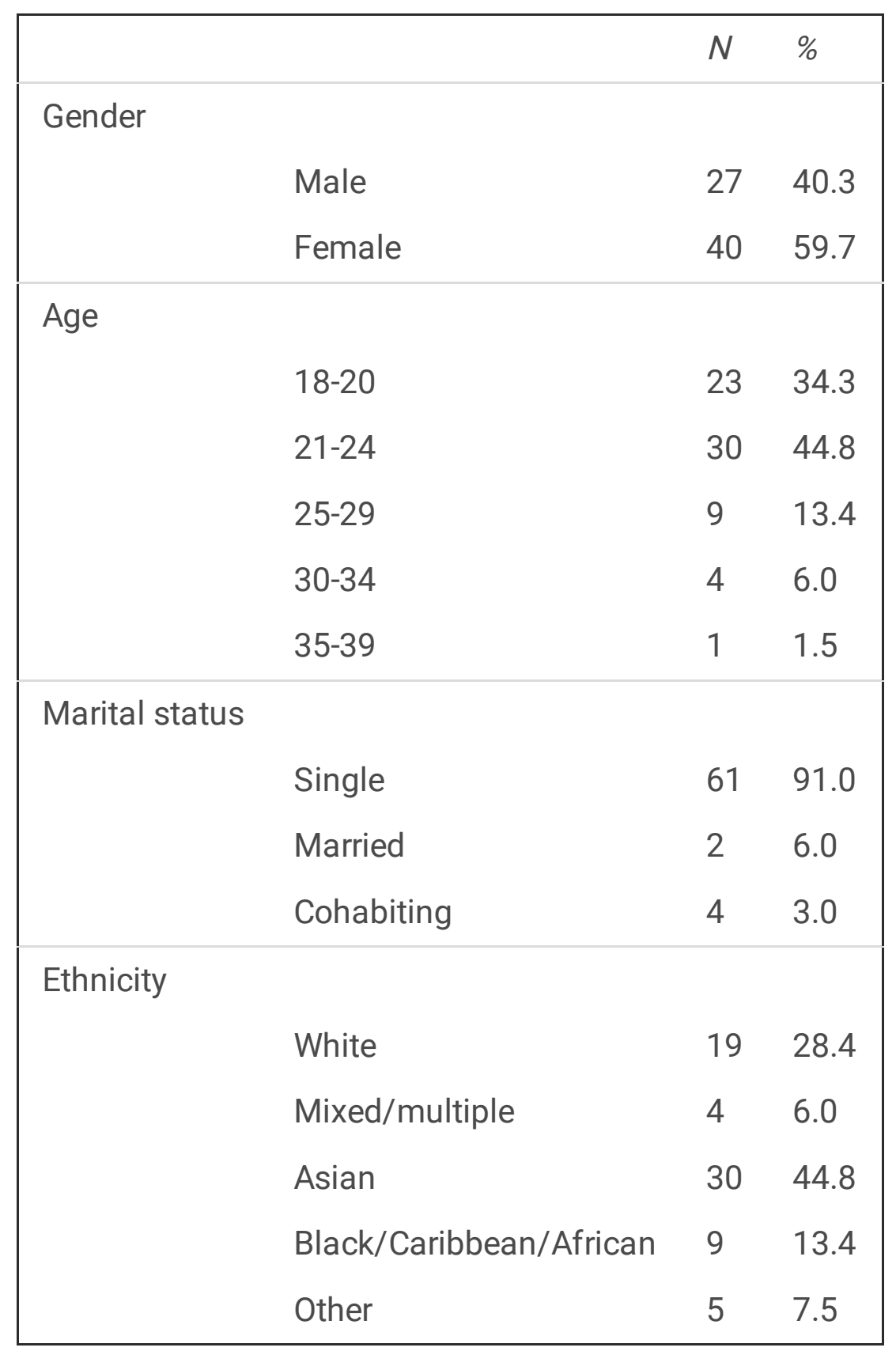

Perceived Stress: Prior to examinations, students were asked to provide a stress rating on a scale from 010 , with 0 representing no stress at all, 5 representing moderate stress, and 10 representing stress as bad as it could be. 
Salivary Cortisol: Participants were instructed to place a cotton swab underneath their tongue for one minute and then transfer the swab to a centrifugation tube with a filter insert. Immediately following collection, samples were spun down for 15 minutes at 25,000 RPM in a centrifuge held at $4 \mathrm{C}$ before being frozen at $-800 \mathrm{C}$ until analysis. Analysis was conducted by ELISA as per the manufacturer's instructions (cat \# 1-3002, Salimetrics, Carlsbad, California, USA) for salivary cortisol measurement.

\section{Five Facet Mindfulness Questionnaire-Short Form (FFMQ-SF; Bohlmeijer, ten Klooster, Fledderus, Veehof,}

\& Baer, 2011): The FFMQ-SF consists of 24 items scored on five-point Likert scales ranging from 1 (never/rarely true) to 5 (very often/always true). It measures five factors representing elements of mindfulness: observing, describing, acting with awareness, non-judging of inner experience, and nonreactivity to inner experience, thus analyses can demonstrate which skills are important predictors of symptom reduction. Facet scores range from 5 to 25, apart from the facet of observing, which ranges from 4 to 20 . The total maximum score on the FFMQ-SF is therefore 120, with higher scores indicating greater levels of mindfulness. The FFMQ-SF has strong psychometric characteristics, including good reliability with alpha coefficients above $a=0.70$ for all facets The FFMQ-SF demonstrated a reliability of $\mathrm{a}=0.70$ in the present study indicating good reliability [26].

\section{Statistical Methods}

Data from two published meta-analyses of research involving the assessment of outcome measures in association with mindfulness $[27,28]$ concluded that studies involving mindfulness produce mean effect sizes of 0.59 and 0.54 , respectively (considered medium sizes, according to Cohen's (1988) criteria [29]. Based on this, an a priori power calculation was undertaken to establish the sample size required; based on a medium effect size $=0.50$, alpha $=0.05$ and confidence level $=0.95$, the sample size required was $\mathrm{n}$ $=45$, protecting against Type I error. Data were analyzed using Prism 9.1.2. 26 .

Data were initially examined for distribution normality and outliers. Baseline and pre-exam salivary cortisol concentration and Perceived Stress scores are presented as means \pm SEM standard error and statistically compared by Student's t-test. Correlations were calculated between baseline measures and end point measures by Spearman's rank correlation test.

\section{Ethical Considerations}

This study was approved by the University School of Science and Medicine Ethics Committee. All students gave their prior written consent for participation in the study and publication of results. So that the data collected were valid and therefore reflective of real life stressors in a medical students' life, written examinations were utilised as stressors for this study. However, efforts were made to minimise the time commitment required of participants before examinations, and to ensure that participation in the study did not induce further stress, nor adversely affect examination performance. In line with this, participants were briefed prior to taking part in the study, and the time taken to complete assessments before examinations was reduced to a maximum of ten minutes. 


\section{Results}

\section{Perceived Stress and salivary cortisol concentrations are increased in the preexam situation}

A paired-samples t-test was conducted to compare perceived stress in the non-stressful condition of group work and the stressful examination condition. There was a significant difference in the scores, indicating that examination conditions are perceived as significantly more stressful than group work conditions $(P<0.0001$, figure $1 A)$. This was confirmed by salivary cortisol levels, which were significantly increased in the examination condition compared with group work $(P<0.0001$, figure $1 B)$.

\section{Exam performance negatively correlates with salivary cortisol levels between baseline and preexam time points}

No significant correlations were found between exam results, perceived stress scores or salivary cortisol concentration at either baseline or pre-exam, although the weak negative correlation between exam performance and salivary cortisol (rho between -0.2 and -0.4 ) had a $\mathrm{P}=0.054$ (table 2). The change in each individual's salivary cortisol concentration had a significant moderate negative correlation with exam score (rho between 0.4 and $0.7, \mathrm{P}<0.0001$, table 2 ) indicating that the greater the change in cortisol levels between group work and pre-exam conditions, the poorer the exam performance.

Table 2. The effect of stress on exam performance.

\begin{tabular}{|lllllll|}
\hline & $\begin{array}{l}\text { Exam } \\
\text { score }\end{array}$ & $\begin{array}{l}\text { Exam score } \\
\text { vs. } \\
\text { Baseline } \\
\text { salivary } \\
\text { cortisol }\end{array}$ & $\begin{array}{l}\text { Exam score } \\
\text { vs. pre- } \\
\text { exam } \\
\text { perceived } \\
\text { stress }\end{array}$ & $\begin{array}{l}\text { Exam score } \\
\text { vs. pre- } \\
\text { exam } \\
\text { salivary } \\
\text { perceived } \\
\text { stress }\end{array}$ & $\begin{array}{l}\text { Exam score } \\
\text { vs. change } \\
\text { in perceived } \\
\text { stress }\end{array}$ & $\begin{array}{l}\text { Exam score } \\
\text { vs. change } \\
\text { in salivary } \\
\text { cortisol }\end{array}$ \\
\hline $\begin{array}{l}\text { Spearman's } \\
\text { r }\end{array}$ & -0.037 & -0.201 & 0.006 & 0.236 & 0.005 & -0.483 \\
\hline P value & 0.769 & 0.103 & 0.959 & 0.054 & 0.970 & $<0.0001$ \\
\hline
\end{tabular}

Spearman's correlation of exam score with perceived stress scores and salivary cortisol levels.

\section{Mindfulness correlates positively with exam performance and negatively with the change in salivary cortisol concentration under preexam stressful conditions}

Exam scores showed a moderate, significant positive correlation with the students' FFMQ total mindfulness score $(r=0.431, P<0.001$, table 3$)$. Exam scores also showed a significant positive correlation with the students' FFMQ acting with awareness score. The FFMQ total mindfulness score also had a significant negative correlation with the change in salivary cortisol concentration from between the baseline, non-stressed sample and the stressful, pre-exam sample $(r=-0.247, P<0.05)$. The pre-exam 
salivary cortisol concentration also had a significant, negative correlation with the FFMQ non-judging score.

Table 3. The effect of mindfulness practice on exam score and stress.

\begin{tabular}{|c|c|c|c|c|c|c|}
\hline & $\begin{array}{l}\text { FFMQ } \\
\text { Total } \\
\text { mindfulness } \\
\text { score }\end{array}$ & $\begin{array}{l}\text { FFMQ } \\
\text { Observing } \\
\text { score }\end{array}$ & $\begin{array}{l}\text { FFMQ } \\
\text { Describing } \\
\text { score }\end{array}$ & $\begin{array}{l}\text { FFMQ Acting } \\
\text { with } \\
\text { awareness } \\
\text { score }\end{array}$ & $\begin{array}{l}\text { FFMQ } \\
\text { Non- } \\
\text { judging } \\
\text { score }\end{array}$ & $\begin{array}{l}\text { FFMQ } \\
\text { Non- } \\
\text { reactivity } \\
\text { score }\end{array}$ \\
\hline \multirow[t]{2}{*}{ Exam score } & $r=0.431$ & $r=0.189$ & $r=-0.181$ & $r=0.354$ & $r=0.187$ & $r=0.391$ \\
\hline & $P=0.0003$ & $P=0.125$ & $P=0.144$ & $P=0.003$ & $P=0.131$ & $P=0.001$ \\
\hline \multirow{2}{*}{$\begin{array}{l}\text { Baseline } \\
\text { perceived } \\
\text { stress }\end{array}$} & $r=0.248$ & $r=0.092$ & $r=-0.235$ & $r=0.118$ & $r=-0.081$ & $r=0.161$ \\
\hline & $P=0.043$ & $P=0.459$ & $P=0.056$ & $P=0.340$ & $P=0.514$ & $P=0.193$ \\
\hline \multirow{2}{*}{$\begin{array}{l}\text { Baseline } \\
\text { salivary } \\
\text { cortisol }\end{array}$} & $r=0.093$ & $r=0.223$ & $r=0.053$ & $r=0.075$ & $r=-0.029$ & $r=0.011$ \\
\hline & $P=0.455$ & $P=0.070$ & $P=0.669$ & $P=0.544$ & $P=0.819$ & $P=0.928$ \\
\hline \multirow{2}{*}{$\begin{array}{l}\text { Pre-exam } \\
\text { perceived } \\
\text { stress }\end{array}$} & $r=0.111$ & $r=0.004$ & $r=-0.019$ & $r=0.128$ & $r=0.045$ & $r=0.095$ \\
\hline & $P=0.370$ & $P=0.977$ & $P=0.877$ & $P=0.304$ & $P=0.715$ & $P=0.444$ \\
\hline \multirow{2}{*}{$\begin{array}{l}\text { Pre-exam } \\
\text { salivary } \\
\text { cortisol }\end{array}$} & $r=-0.147$ & $r=0.107$ & $r=0.154$ & $r=-0.052$ & $r=-0.244$ & $r=-0.165$ \\
\hline & $P=0.235$ & $P=0.391$ & $P=0.214$ & $P=0.674$ & $P=0.046$ & $P=0.183$ \\
\hline \multirow{2}{*}{$\begin{array}{l}\text { Change in } \\
\text { perceived } \\
\text { stress }\end{array}$} & $r=-0.203$ & $r=-0.076$ & $r=0.228$ & $r=-0.062$ & $r=0.097$ & $r=-0.122$ \\
\hline & $P=0.099$ & $P=0.542$ & $P=0.063$ & $P=0.617$ & $P=0.437$ & $P=0.325$ \\
\hline \multirow{2}{*}{$\begin{array}{l}\text { Change in } \\
\text { salivary } \\
\text { cortisol }\end{array}$} & $r=-0.247$ & $r=-0.125$ & $r=0.122$ & $r=-0.174$ & $r=-0.202$ & $r=-0.163$ \\
\hline & $P=0.044$ & $P=0.313$ & $P=0.327$ & $P=0.160$ & $P=0.100$ & $P=0.187$ \\
\hline
\end{tabular}

Spearman's correlation of exam score and stress markers with FFMQ mindfulness scores.

\section{Discussion}

In our opportunistic study, healthy medical students were exposed to an objectively stressful situation of an exam, and relationships between exam performance mindfulness, perceived stress and serum cortisol in response to this and a non-stressful control event, were examined. This study therefore explored whether participants' changes in subjective experience were associated with stress-related salivary concentrations of serum cortisol and exam performance, and whether mindfulness experience could buffer these effects. The present study demonstrated that exam conditions elevated cortisol, and increased cortisol was correlated with a reduced exam performance. Importantly, students who scored more highly in the FFMQ questionnaire displayed reduced levels of salivary cortisol immediately prior to exams and performed better in the exam itself than those with lower scores on the FFMQ. This suggests 
that there exists an important relationship between mindfulness and biomarkers of stress, which may manifest into improved assessment outcomes potentially through healthier, more adaptive coping and stress management strategies.

These findings align with a study that found that greater perceived stress before an exam was associated with greater levels of B-cells (another important clinical marker of immune activity that may increase chronic tissue inflammation) [25]. However, in contrast to the present study, that study found no further associations between biomarkers of distress (including serum cortisol, among others) and mindfulness. Such conflicting evidence demands the need for larger, randomized trials to clearly establish the relationships between variables examined in the present study, and other work in this field.

A novel strength of the present study is the ability to explore the nuance within the measure of mindfulness and individual facets, with results demonstrating that exam scores showed a significant positive correlation with the students' FFMQ acting with awareness score, whilst the pre-exam salivary cortisol concentration had a significant, negative correlation with the FFMQ non-judging score. This suggests that these particular facets of mindfulness, acting with awareness (attending to what is happening in the present) and non-judging (taking a non-evaluative stance toward internal thoughts and feelings), are particularly important skills in managing exam-related stress. Indeed, a non-judgmental stance towards stress-inducing circumstances such as exams may make students less likely to engage with negative appraisals and catastrophic thinking surrounding the experience, and as such become less prone to increased perceived stress and cortisol excretion. Likewise, acting with awareness may mitigate stress through the prioritization of focus on the present, which may reduce engagement in hypervigilance and attention paid to negative appraisals [30]. This finding echoes that of previous work in which both of these factors were found to mitigate anxiety in medical students [31], as well as being associated with lower depression severity in general populations, and represents potentially important areas for future work to focus on improving.

The results of the present study support the suggestion that the integration of mindfulness training as a supportive mechanism in medical school (as suggested by the UK General Medical Council) is warranted and of value. Indeed, multiple medical schools already provide mindfulness resources to support their students [32]. Others have furthered this by integrating mindfulness into their curriculum, such as Monash University in Australia, with studies showing decreased depression and hostility, even pre-exam time, as a result [14]. However, being afforded the choice to participate in mindfulness training may influence its effects. Students were less satisfied with mandatory mindfulness training, compared to voluntary sessions, which suggests that integration into the curriculum may not always be the most effective approach [33]. Further research is required to determine the more efficacious ways to support mindfulness practice in medical students in order to maximise potential benefits and minimise dissatisfaction or adverse effects. Institutional support is crucial to the successful implementation of mindfulness and this can take many forms at multiple levels [34]. It is recommended that individual schools consult their student and staff body and elect to offer mindfulness training in ways that best suit their own curriculum and student need and demand. Regardless of the approach medical schools may 
opt to take, the present study provides further evidence of the potential that mindfulness could offer in improving stress management and academic success.

\section{Limitations And Future Research}

Cortisol is a reliable biomarker of stress [18](Bozovic, 2013), however it is not only secreted in response to stressors, but in a regular diurnal fashion which could be a confounding limitation to the study. The peak cortisol level occurs 30 min after waking, and is back to the daily average after 1 hour and stays in the average range for several hours [17](Elder et al. 2014). Accordingly, the morning exam time and sampling likely occurred at a time when the diurnal variation is at its most stable, as has been demonstrated on other studies [17](Elder et al., 2014). Further replication work could add value to our understanding of the role of stress, cortisol, and mindfulness in exam performance by exploring variations in cortisol throughout the day of an assessment to establish the extent to which this variable, and the extent of change in cortisol, are associated with mindfulness and exam performance. This would add depth to our understanding of the role of mindfulness as a supportive mechanism in managing stress in medical students. Additionally, whilst our controlled pre-post analysis is a strength of the present study, further work could explore the directionality of these relationships in a longitudinal manner. Longitudinal work would be beneficial in establishing the extent to which further training in mindfulness can improve perceived stress, reduce cortisol production in response to stressful events, and improve performance in academic assessments such as medical school exams, and beyond in medical practice.

\section{Conclusions}

There is an important relationship between mindfulness and biomarkers of stress, which may manifest into improved assessment outcomes potentially through healthier, more adaptive coping and stress management strategies. A novel strength of the present study is the identification of individual facets of mindfulness associated with stress and exam performance (acting with awareness and non-judging) suggesting that these may be important targets for improving medical students' stress management and exam performance.

\section{List Of Abbreviations}

FFMQ Five Facet Mindfulness Questionnaire

FFMQ-SF Five Facet Mindfulness Questionnaire - Short Form

\section{Declarations}

\section{Disclosure Statement}

The study also involved the handling of saliva, which is protected under the Human Tissues Act (2004) and may be bio hazardous material, which was handled in accordance with the Human Tissues Act. 
The authors declare that there are no conflicts of interest, and agree to the publication of this article. There are no awarding grant bodies to be acknowledged.

\section{Acknowledgements}

The authors wish to acknowledge the University of Buckingham Medical School students for participating in the study.

\section{Funding}

There is no funding documentation relevant to this study.

\section{Availability of data and materials}

The data are available to all interested researchers upon request. Please contact the corresponding author.

\section{Authors' contributions}

$\mathrm{CS}$ and $\mathrm{JH}$ led the development of the study. Data were collected by JH with support from student researchers. Statistical analyses were performed by JH and CS. The manuscript was written and approved by $\mathrm{JH}$ and CS.

\section{Authors' information}

$\mathrm{JH}$ is a senior lecturer in Psychology in the Department of Psychology, Manchester Metropolitan University.

CS is the Phase 1 Lead at Aston Medical School, Aston University.

\section{Ethics approval and consent to participate}

This study was approved by the University School of Science and Medicine Ethics Committee. All students gave their prior written consent for participation in the study.

\section{Consent for publication}

All students gave their prior written consent for publication of results.

\section{Competing interests}

The authors declare that they have no competing interests.

\section{References}


1. Kulsoom B, Afsar NA. Stress, anxiety, and depression among medical students in a multiethnic setting. Neuropsychiatr Dis Treat. 2015; 11: 1713-1722. Doi: https//doi.org/ 10.2147/NDT.S83577

2. Lyndon MP, Strom JM, Alyami HM, Yu TC, Wilson NC, Singh PP, Lemanu DP, Yielder J, Hill AG. The relationship between academic assessment and psychological distress among medical students: a systematic review. Perspect Med Educ. 2014;3:405 - 18. Doi: https//doi.org/10.1007/s40037-0140148-6

3. Rotenstein LS, Huckman RS, Wagle NW. Making Patients and Doctors Happier - The Potential of Patient-Reported Outcomes. N Engl J Med. 2017;377:1309-1312. Doi: https//doi.org/ 10.1056/NEJMp1707537

4. Pacheco JP, Giacomin HT, Tam WW, Ribeiro TB, Arab C, Bezerra IM, Pinasco GC. Mental health problems among medical students in Brazil: a systematic review and meta-analysis. Braz J Psychiatry. 2017;39:369-378. Doi: https//doi.org/10.1590/1516-4446-2017-2223

5. Dyrbye LN, Thomas MR, Shanafelt TD. Systematic review of depression, anxiety, and other indicators of psychological distress among U.S. and Canadian medical students. Acad Med. 2006;81:354-73. doi: https://doi.org/10.1097/00001888-200604000-00009

6. Sohail N. Stress and academic performance among medical students. J Coll Physicians Surg Pak. 2013;23:67-71. Available at: https://www.jcpsp.pk/archive/2013/Jan2013/15.pdf

7. Kötter T, Wagner J, Brüheim L, Voltmer E. Perceived medical school stress of undergraduate medical students predicts academic performance: an observational study. BMC Med Educ. 2017;17(1):256. https://doi.org/10.1186/s12909-017-1091-0

8. McEwan K, Gilbert P, Duarte J. An exploration of competitiveness and caring in relation to psychopathology. Br J Clin Psychol. 2012. 51(1):19-36. Doi: https//doi.org/10.1111/j.20448260.2011.02010.x

9. Neumann M, Edelhäuser F, Tauschel D, Fischer MR, Wirtz M, Woopen C, Haramati A, Scheffer C. Empathy decline and its reasons: a systematic review of studies with medical students and residents. Acad Med. 2011;86:996-1009. Doi: https//doi.org/ 10.1111/j.2044-8260.2011.02010.x

10. Hojat $M$, Vergare $M$, Isenberg $G$, Cohen $M$, Spandorfer J. Underlying construct of empathy, optimism, and burnout in medical students. Int J Med Educ. 2015; 6:12-6. doi: https://doi.org/10.5116/ijme.54c3.60cd

11. Sandover S, Jonas-Dwyer D, Marr T. Graduate entry and undergraduate medical students' study approaches, stress levels and ways of coping: a five year longitudinal study. BMC Med Educ. 2015;15:5. Doi: https//doi.org/10.1186/s12909-015-0284-7

12. Stewart SM, Lam TH, Betson CL, Wong CM, Wong AM. A prospective analysis of stress and academic performance in the first two years of medical school. Med Educ. 1999;33:243 - 50. Doi: https://doi.org/ 10.1046/j.1365-2923.1999.00294.x.

13. Phang CK, Mukhtar F, Ibrahim N, Keng SL, Mohd Sidik S. (2015). Effects of a brief mindfulness-based intervention program for stress management among medical students: the Mindful-Gym randomized 
controlled study. Adv Health Sci Educ Theory Pract. 2015;20:1115-34. Doi:

https//doi.org/10.1007/s10459-015-9591-3

14. Hassed C, de Lisle S, Sullivan G, Pier C. Enhancing the health of medical students: outcomes of an integrated mindfulness and lifestyle program. Adv Health Sci Educ Theory Pract. 2009;14:387-98. doi: https://doi.org/10.1007/s10459-008-9125-3

15. Lampe LC, Müller-Hilke B. Mindfulness-based intervention helps preclinical medical students to contain stress, maintain mindfulness and improve academic success. BMC Med Educ 21, 145 (2021). https://doi.org/10.1186/s12909-021-02578-y

16. Daya Z, Hearn JH. Mindfulness interventions in medical education: A systematic review of their impact on medical student stress, depression, fatigue and burnout. Med Teach. 2018;40:146-153. doi: https://doi.org/10.1080/0142159X.2017.1394999

17. Elder GJ, Wetherell MA, Barclay NL, Ellis JG. The cortisol awakening response - Applications and implications for sleep medicine. Sleep Medicine Reviews. 2014;18:215-224. Doi: https://doi.org/10.1016/j.smrv.2013.05.00

18. Bozovic D, Racic M, Ivkovic N. Salivary cortisol levels as a biological marker of stress reaction. Med Arch. 2013;67:374-7. Doi: https://doi.org/10.5455/medarh.2013.67.374-377

19. McEwen BS. Physiology and neurobiology of stress and adaptation: central role of the brain. Physiol Rev. 2007;87:873-904. Doi: https//doi.org/10.1152/physrev.00041.2006

20. Dinkel K, MacPherson A, Sapolsky RM. Novel glucocorticoid effects on acute inflammation in the CNS. J Neurochem. 2003;84:705-716. doi: https://doi.org/10.1046/j.1471-4159.2003.01604.x

21. McEwen BS, Chattarji S. Molecular mechanisms of neuroplasticity and pharmacological implications: the example of tianeptine. Eur Neuropsychopharmacol. 2004;14:S497-S502. Doi: https//doi.org/ 10.1016/j.euroneuro.2004.09.008

22. Joëls M. Corticosteroid effects in the brain: U-shape it. Trends Pharmacol Sci. 2006;27:244-250. Doi: https://doi.org/10.1016/j.tips.2006.03.007

23. Lupien SJ, de Leon M, de Santi S, Convit A, Tarshish C, Nair NPV, Thakur M, McEwen BS, Hauger RL, Meaney MJ. Cortisol levels during human aging predict hippocampal atrophy and memory deficits. Nature Neurosci. 1998;1:69-73. Doi: https//doi.org/10.1038/271

24. Turakitwanakan W, Mekseepralard C, Busarakumtragul P. Effects of mindfulness meditation on serum cortisol of medical students. J Med Assoc Thai. 2013;96:S90-95.

25. Turner L, Galante J, Vainre M, Stochl J, Dufour G, Jones PBD. Immune dysregulation among students exposed to exam stress and its mitigation by mindfulness training: findings from an exploratory randomised trial. Clinical Trial Sci Rep. 2020;10:5812. Doi: https://doi.org/ 10.1038/s41598-02062274-7

26. Bohlmeijer E, ten Klooster PM, Fledderus M, Veehof M, Baer R. Psychometric properties of the five facet mindfulness questionnaire in depressed adults and development of a short form. Assessment. 2011;18:308-320. Doi: https://doi.org/10.1177/1073191111408231 
27. Baer RA. (2003) Mindfulness Training as a Clinical Intervention: A Conceptual and Empirical Review. Clinical Psychology: Science and Practice. 2003;10:125-143. Doi: http://dx.doi.org/10.1093/clipsy.bpg015

28. Grossman P, Niemann L, Schmidt S, Walach H. Mindfulness-based stress reduction and health benefits. A meta-analysis. J Psychosom Res. 2004;57:35-43. doi: https://doi.org/10.1016/S00223999(03)00573-7

29. Cohen J. Statistical Power Analysis for the Behavioral Sciences. 2nd Edition. Hillsdale: Lawrence Erlbaum; 1988

30. Vanden Bogaerde A, Derom E, De Raedt R. Increased interoceptive awareness in fear of flying: Sensitivity to suffocation signals. Behaviour Research and Therapy. 2011;49:427-432. Doi: https://doi.org/10.1016/j.brat.2011.03.011

31. Fino E, Martoni M, Russo PM. Specific mindfulness traits protect against negative effects of trait anxiety on medical student wellbeing during high-pressure periods. Adv in Health Sci Educ. 2021; Doi: https://doi-org.mmu.idm.oclc.org/10.1007/s10459-021-10039-w

32. Barnes N, Hattan P, Black DS, Schuman-Olivier Z. An Examination of Mindfulness-Based Programs in US Medical Schools. Mindfulness. 2017;8:489-494. Doi: https://doi.org/10.1007/s12671-016-06238

33. Aherne D, Farrant K, Hickey L, Hickey E, McGrath L, McGrath D. Mindfulness based stress reduction for medical students: optimising student satisfaction and engagement. BMC Med Educ. 2016;16:209. doi: https://doi.org/10.1186/s12909-016-0728-8

34. Rac T, Chakravarti A. Eight ways to get a grip on implementing mindfulness sessions in medical schools. Can Med Educ J. 2020;11:e130-e134. Doi: https://doi.org/ 10.36834/cmej.57011

\section{Figures}
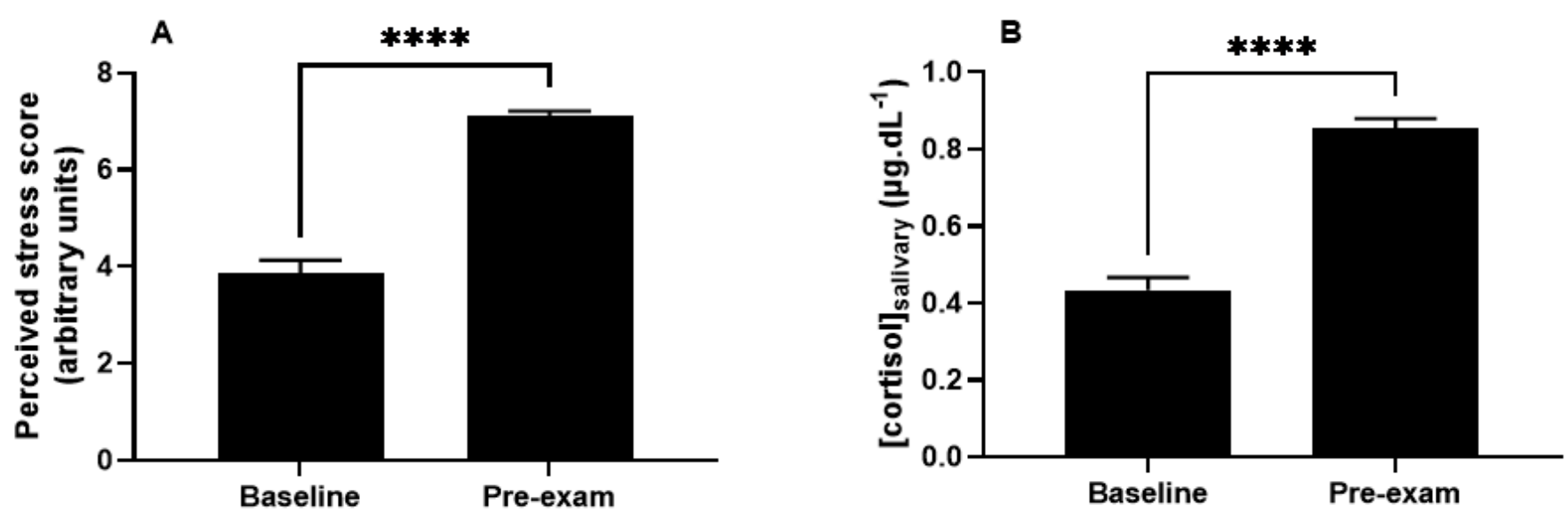

Figure 1 
The effect of exam conditions on stress Baseline and pre-exam levels of perceived stress (A) and salivary cortisol concentration (B). $\mathrm{N}=67 .{ }^{\star \star \star *} \mathrm{P}<0.0001$. 